\title{
Using weight decision for decreasing the price of anarchy in bin packing games
}

\author{
Gyorgy Dosa, ${ }^{*}$ Hans Kellerer, ${ }^{\dagger}$ Zsolt Tuza ${ }^{\ddagger}$ \\ Latest update on 17 August 2018
}

\begin{abstract}
A selfish bin packing game is a variant of the classical bin packing problem in a game theoretic setting. In our model the items have not only a size but also a nonnegative weight. Each item plays the role of a selfish agent, and any agent/item pays some cost for being in a bin. The cost of a bin is 1 , and this cost is shared among the items being in the bin, proportionally to their weight. A packing of the items into bins is called a Nash equilibrium if no item can decrease its cost by moving to another bin. In this paper we present two different settings for the weights which provide better values for the price of anarchy $(\mathrm{PoA})$ than previous settings investigated so far. The improved PoA is not bigger than $16 / 11 \approx 1.4545$. Moreover, we give a general lower bound for the price of anarchy which holds for all possible choices of the weights.
\end{abstract}

Keywords: Selfish bin packing, price of anarchy, algorithmic game theory

\section{Introduction}

The bin packing problem [11] is one of the classical combinatorial optimization problems. We are given a set $I=\{1,2, \ldots, n\}$ of $n$ items, the size of item $i$ is $s_{i} \in(0,1]$. The goal is to pack the items into a minimum number of bins, so that any bin can contain only items with total size at most 1 . For a survey on approximation algorithms for bin packing problems see [3].

\footnotetext{
* Department of Mathematics, University of Pannonia, H-8200 Veszprém, Egyetem u. 10, Hungary. E-mail: dosagy@almos.vein.hu

† Institut für Statistik und Operations Research, Universität Graz, Universitätsstraße 15, 8010 Graz, Austria. E-mail: hans.kellerer@uni-graz.at

$¥$ Alfréd Rényi Institute of Mathematics, Hungarian Academy of Sciences, H-1053 Budapest, Reáltanoda u. 13-15; and Department of Computer Science and Systems Technology, University of Pannonia, H-8200 Veszprém, Egyetem u. 10, Hungary. E-mail: tuza@dcs.uni-pannon.hu.
} 
In a selfish bin packing game (in our model) an item $i$ has not only a size but also a positive weight, denoted by $g_{i}$. Each item plays the role of a selfish agent, and any agent/item pays some cost for being in a bin. The cost of a bin is 1 , and this cost is shared among the items being in the bin, proportionally to their weight $g_{i}$; i.e., any item $i$ packed into a bin (with total size at most 1 ) pays the $\operatorname{cost}^{1} g_{i} / g(B)$ where $g(B)$ is the total weight of the items in the bin. Analogously, we denote by $s(B)$ the total size of items being packed into this bin, also called level of the bin.

Throughout this paper we assume that all weights are positive, i.e., $g_{i}>$ 0 holds for all $1 \leq i \leq n$. This condition excludes situations where certain priviliged items make extra profit by increasing the costs of others.

Each item is interested in decreasing its own cost. So, if an item fits into another bin, and moving there the cost of this item will be strictly smaller than before, we call this move an improving step.

A Nash equilibrium ( $N E$, for short) is a packing where an improving step, as described above, does not exist for any item. Such a $N E$ is also called a stable packing. The following simple assertion states that a $N E$ packing is reached from any initial (valid) packing after finitely many selfish steps, also in a more general model.

Observation 1 Consider a cost-sharing mechanism in which the input consists of items $i$ with given size $s_{i}$ and weight $g_{i}(i=1, \ldots, n)$, with the following property:

( $\star$ If in a valid packing an item $i$ moves from a bin $B$ to another bin $B^{\prime}$ where it fits and the total weight in $B^{\prime}$ becomes larger than the original weight in $B$, then $i$ should pay less in $B^{\prime}$; but its cost does not decrease if the modified $g\left(B^{\prime}\right)$ remains at most the current $g(B)$.

Then, starting from any initial packing, the game terminates in a NE after a finite number of selfish moves.

Proof. Define a potential function $\psi$ for all feasible packings $\mathcal{B}$ as

$$
\psi(\mathcal{B})=\sum_{B \in \mathcal{B}}(g(B))^{2}
$$

where the summation is taken over all used bins $B$. Under a selfish move of item $i$ the contents of two bins, say $B_{j}$ and $B_{k}$, are modified to $B_{j}^{\prime}$ and $B_{k}^{\prime}$ such that

$g\left(B_{j}^{\prime}\right)+g\left(B_{k}^{\prime}\right)=g\left(B_{j}\right)+g\left(B_{k}\right) \quad$ and $\quad\left|g\left(B_{j}^{\prime}\right)-g\left(B_{k}^{\prime}\right)\right|>g_{i}>\left|g\left(B_{j}\right)-g\left(B_{k}\right)\right|$.

\footnotetext{
${ }^{1}$ One can create many other types of cost-sharing mechanisms beside this linear function, as it happens in real life, too. The cost distribution $g_{i} / g(B)$ is relevant when the relative cost is expressible by one single number.
} 
It follows that the value of $\psi$ increases after every selfish move, and consequently no $\mathcal{B}$ can occur more than once during a game. Since each input admits only finitely many possible packings, the process surely terminates.

Property $(\star)$ is definitely more general than the cost-sharing rule $g_{i} / g(B)$. For example if $g_{1}, \ldots, g_{k}$ are the weights of items packed in $B$, and instead of 1 their total cost is $\sqrt{g_{1}+\cdots+g_{k}}$, the cost distribution proportional to the $g_{i}$ among those items satisfies $(\star)$. A priciple like that occurs in many real-life situations, from the "pay 2, buy 3" promotions in stores to the shared renting of an apartment which is more economical for the individual than renting a separate apartment alone.

Note that in case of a $N E$ packing, the number of used bins can be much larger than necessary for packing the items in an optimal solution for classical bin packing. Therefore, it is interesting to compute the price of anarchy $(P o A)$ [12] of the game which is in general given by the maximum ratio between the maximum social cost of any $N E$, and the minimum social cost of any situation. In our case, it is defined as

$$
P o A=\limsup _{N \rightarrow \infty}\left(\sup _{I} \max _{N E \in \mathcal{N} \mathcal{E}(I)}\left\{\frac{N E(I)}{O P T(I)} \mid O P T(I)=N\right\}\right),
$$

where $I$ is an arbitrary (finite) set of items, $\mathcal{N E}(I)$ is the set of all Nash equilibria for $I, N E(I)$ is the number of used bins in a Nash equilibrium packing $N E$ of the items, and $O P T(I)$ denotes the number of bins used in an optimal packing of the items. If it is clear from the context, we will write $N E$ instead of $N E(I)$ and $O P T$ instead of $O P T(I)$, respectively.

Next, we provide a short review about selfish bin packing games. The first such game was defined by Bilò [1] for $g_{i}=s_{i}$. Consequently, the cost of an item $i$ for being in bin $B$ is $s_{i} / s(B)$ in this case. Bilò proved that $8 / 5 \leq P o A \leq 5 / 3$ holds in this game. Epstein and Kleiman [8] tightened the gap between the lower and upper bound to $1.6416<P o A<1.6428$.

Ma et al. [13] defined another model. If there are $k$ items in a bin, each item in this bin pays $1 / k$ cost for being in this bin. This corresponds to the case $g_{i}=1$. It is shown that any $N E$ packing is the output of a run of the First Fit $(F F)$ algorithm (which packs the items according to some given list, and the next item is always packed into the first bin where it fits [11]). From this, it directly follows that the $P o A$ of this version of the game is at most 1.7. Later Dosa and Epstein [4] gave a more exact estimation, showing that the $P o A$ is in the interval $(1.6966,1.6994)$.

There are also many other results which correspond to the models listed above; see, e.g., $[8,9,10,16]$. In fact there is also a model in the literature which generalizes all previous selfish bin packing games [15]. 
Returning to our game, we have seen that the two different models (sharing the cost proportionally to the sizes, or equally, regardless of item sizes) give two different $P o A$ values. For the former this is at most 1.6428 , but for the latter it is bigger than $1.6966[8,4]$. Note that both these cost-sharing mechanisms are special cases of our more general model, where the cost is distributed proportionally to the weights of the items. It means that the weight matters. Notice that in [4] it is proven that by any choice of the weights, the $P o A$ is at most 1.7, and there exists a choice where this value is exactly 1.7 .

In this work we attack the problem from the opposite side. Observe that PoA is a cost which has to be paid by a community because its members behave in a selfish non-cooperative way. For this reason the community may try to define weights so as to decrease the price of anarchy. By this motivation, we raise and investigate the following question.

Problem 2 How should one define the weights for the items, so that the PoA will be as small as possible? What is the minimum or infimum of PoA achievable by suitable weight setting?

This problem - in a more general context - sounds as follows: Can one determine the rules of a structure ${ }^{2}$ (in our case it is the current game) in such a way that the behavior of participants will be advantageous for the whole community? If the answer is yes, how to do this? In the language of games: Can one construct a game where the $P o A$ is very small?

A recent work in this direction is [17] which defines a cost-sharing mechanism with $P o A \approx 1.467$. Another similar work is [2] where the $P o A$ is between 1.47407 and 1.4748. A third paper on a mechanism with larger $P o A=3 / 2$ (although fast convergence to $N E$ ) is [14]. This latter result motivates the following variant of Problem 2.

Problem 3 What is the minimum or infimum of PoA achievable by suitable weight setting with the additional condition that a $N E$ is reached in polynomial time from every initial packing?

We are interested in defining a game with a $P o A$ smaller than that in all earlier results, i.e., we concentrate on Problem 2 without the time constraint of Problem 3.

We will consider two settings for the weights in this paper. In the first one, in most cases the weight of an item is equal to its size, except that the items having sizes above one half get larger weight, namely their weight will be set to 1 . For this special setting we will see that the $P o A$ is already at most 1.5.

\footnotetext{
${ }^{2}$ An existing example on the large scale is that different types of products include different percentages of VAT in their price, hence orienting the consumption habits of people to some extent.
} 
Although the $P o A$ in this case is above 1.4646, the principle of the construction provides a frame for later improvements and hence in this aspect it is crucial. On its basis we define and investigate a more complicated setting, where the $P o A$ is at most $16 / 11 \approx 1.4545$, and at least 1.4528 .

It is important to note that the $P o A$ cannot be very small, namely we shall prove that in a wide class of games (in not only those with weight-driven rules) it can never be smaller than $4 / 3$.

The paper is structured as follows. In Section 2 we present a general lower bound for the price of anarchy. Section 3 contains the first simple setting which has $P o A$ of at most 3/2. In Section 4 we present the more complicated setting with $P o A$ at most 16/11. We finish in Section 5 with short conclusions and open problems.

This work is a substantially extended version of the conference paper [5].

\section{A General Lower Bound}

In this section we present a general lower bound for the $P o A$, which universally is valid no matter how the weights for the items are chosen. The construction is based on the property that no item can move to another bin since it does not fit. In fact the lower bound is valid for games in a more general class, too.

Theorem 4 If the rules of the game do not allow a packed item to open a new bin and move there alone, then the PoA is at least 4/3.

Proof. We make the following construction. Let $n$ be divisible by four, and let $\varepsilon$ be a sufficiently small rational number. We construct a packing into $n$ bins that is a $N E$. Then we show that the items can be packed into $(3 / 4) n+1$ bins proving our claim.

In any $N E$ bin there are two items, a medium item and a small item, denoted by $M_{i}$ and $S_{i}, 1 \leq i \leq n$, respectively. In the first $n / 2$ bins the medium items have the same size, $M_{i}=1 / 3+(2 n-1) \varepsilon$, for $1 \leq i \leq n / 2$, these are the biggest items in our construction. In the other bins the medium items have sizes as follows: $M_{i}=1 / 3+\left(\frac{5}{2} n-i\right) \varepsilon$, for $n / 2+1 \leq i \leq n$ (then $M_{n / 2+1}$ has the same size as the previous medium items, and the sizes of the medium items are slightly decreasing from this point.)

Now we give the sizes of the small items in the bins.

For any $n / 2+1 \leq i \leq n$, let $S_{i}=1 / 3-\left(\frac{3}{2} n-i\right) \varepsilon$. Hence, $M_{i}+S_{i}=2 / 3+n \varepsilon$. Note that the smallest small item among these items is $S_{n / 2+1}=1 / 3-(n-1) \varepsilon$. This is the size of any further small items as well, so $S_{i}=1 / 3-(n-1) \varepsilon$ for $1 \leq i \leq n / 2$. (The biggest small item has size $S_{n}=1 / 3-(n / 2) \varepsilon$.)

Then the levels of the bins are as follows. We have level $1 / 3+(2 n-1) \varepsilon+$ $1 / 3-(n-1) \varepsilon=2 / 3+n \varepsilon$ in the first $n / 2$ bins and also exactly $2 / 3+n \varepsilon$ in the 
last $n / 2$ bins. It is trivial that this packing is a $N E$, as no item fits into another bin (to see this, it is enough to see that the smallest item does not fit into any other bin).

Now we show that the items can be packed into $(3 / 4) n+1$ bins.

We create $n / 2-1$ bins as follows. For any $n / 2+2 \leq i \leq n$, the bin contains $M_{i}$, moreover $S_{i-1}$ and finally $S_{i-n / 2}$. Let us realize that $M_{i}+S_{i-1}=$ $2 / 3+(n-1) \varepsilon$, so after packing these two items in the bin, the remaining space in the bin is just the size of $S_{i-n / 2}$. So these three items fit into a common bin.

The largest medium items are packed pairwise, and so we get $n / 4$ further bins.

There remain one medium item and two small items unpacked (namely $M_{n / 2+1}, S_{n}$ and finally $\left.S_{n / 2}\right)$. We pack them into two further bins and get in total $(n / 2-1)+n / 4+2=(3 / 4) n+1$ bins.

Observation 5 In the previous construction the level of any $N E$ bin is exactly $2 / 3+n \varepsilon$, and the level of any not full optimal bins is exactly $2 / 3-(4 n-2) \varepsilon$.

Since the $P_{o A}$ is computed as a limes superior, it does not matter if a certain property does not hold for a bounded number of bins. Bins for which a certain property does not hold, will be called irregular in the following. The bins which are not irregular are called regular bins.

At the end of this section we provide a simple lemma which will be useful several times.

Lemma 6 Suppose that the level of any $N E$ bin is at least $l>0$ with at most $C$ irregular bins. Then $N E \leq(1 / l) \cdot O P T+C$.

Proof. We have $O P T \geq S \geq l \cdot(N E-C)$ where $S$ denotes the total level of the bins. From this we get $N E \leq(1 / l) \cdot O P T+C$.

\section{Two Levels of Weights: Setting $S 1$}

In this section we will examine a simple setting, denoted as $S 1$, in which the weights of the items are defined as follows. Let the items be called big and small (denoted by $B$ and $S$ ) if their sizes are larger than $1 / 2$, or at most $1 / 2$, respectively. The big items get weight 1 , and each small item gets weight equal to its size. The weights in setting $S 1$ are illustrated in Table 1 . In the following we give lower and upper bounds for the PoA of setting $S 1$.

\subsection{Basic Lower Bound for $S 1$}

As a first step, in this subsection we prove the following lower bound for the PoA of $S 1$. The method will be developed further afterwards, to obtain a stronger estimate. 


\begin{tabular}{|r|c|c|}
\hline Class & Size $s_{i}$ & Weight $g_{i}$ \\
\hline $\mathrm{big}$ & $s_{i}>\frac{1}{2}$ & $g_{i}=1$ \\
\hline small & $s_{i} \leq \frac{1}{2}$ & $g_{i}=s_{i}$ \\
\hline
\end{tabular}

Table 1: Weights for setting $S 1$

Theorem 7 For setting $S 1$ the PoA is at least $\frac{4}{3}+\frac{1}{3} \cdot \sum_{k=1}^{\infty} \frac{1}{3 \cdot 2^{k}-1} \approx 1.4589$.

Proof. We extend our construction from Theorem 4. The original items of that instance, say $I$, are called old items. Let us recall that in the optimum packing of $I$, the $n / 2$ largest medium items are packed pairwise into a set of $n / 4$ bins, which we shall denote by $\mathcal{B}$. The bins in $\mathcal{B}$ have some space left for further items. Let us consider any bin of $\mathcal{B}$. The unused space of such a bin is $1 / 3-(4 n-2) \varepsilon$ by Observation 5 , that is, a bit below $1 / 3$. We will use that space for packing smaller items.

Let $k \geq 4$ be a very large integer. We create a new instance $I^{\prime}$ which augments $I$ by the following new items: $n / 4$ items of size $a_{1}=1 / 6+n \varepsilon, n / 4$ items of size $a_{2}=1 / 12+n \varepsilon$, and so on. In general, we get for any $1 \leq i \leq k$, $n / 4$ new items of size $a_{i}=\frac{1}{3 \cdot 2^{i}}+n \varepsilon$ so that the smallest item $a_{k}$ has size $a_{k}=\frac{1}{3 \cdot 2^{k}}+n \varepsilon$.

We get a packing for the items in $I^{\prime}$ by adding to each bin of $\mathcal{B}$ items of sizes $a_{1}, a_{2}, \ldots, a_{k}$, exactly one item for each size. The total size of the items in any such bin is

$$
1-\frac{1}{3 \cdot 2^{k}}+(k n+4 n-2) \varepsilon \leq 1-\frac{1}{3 \cdot 2^{k}}+2 k n \varepsilon .
$$

We choose $\varepsilon$ such that the items fit together, so let $\varepsilon \leq 1 /\left(6 k n \cdot 2^{k}\right)$. In this way all bins are "almost" completely full.

The optimal packing for $I$ used $(3 / 4) / n+1$ bins. Consequently, the optimal packing for $I^{\prime}$ does also use not more than $(3 / 4) / n+1$ bins.

Now we construct a $N E$ for the instance $I^{\prime}$. Without loss of generality, we suppose that $n$ is divisible by $3 \cdot 2^{k}-1$ for any $1 \leq i \leq k$. The old items are packed as before. In any new bin, items of the same size $a_{i}(i=1, \ldots, k)$ are packed. That means, there are bins containing five $a_{1}$ items, also there are bins containing eleven $a_{2}$ items, and so on. Generally, there are bins containing $3 \cdot 2^{i}-1$ items of type $a_{i}, 1 \leq i \leq k$. It follows that the number of newly created bins is

$$
\frac{n}{4} \cdot \sum_{i=1}^{k} \frac{1}{3 \cdot 2^{i}-1} .
$$


The total number of bins in the packing is

$$
n+\frac{n}{4} \cdot \sum_{i=1}^{k} \frac{1}{3 \cdot 2^{i}-1}
$$

while there is an optimal packing using only $(3 / 4) n+1$ bins. Thus the lower bound follows, once we prove that this packing is a $N E$.

We show that no item can make an improving step. It is trivial that no old item can move as it fits into no other bin. Let us suppose that the bins containing the new items are ordered in nonincreasing order of their levels. Then, we have:

- No new item fits into a bin to the right.

- No new item wants to move to the left.

This completes the proof of the theorem.

\subsection{A More Complex Construction: $P o A>1.46460806$}

Here we give a more involved construction to obtain an improved lower bound for the $\operatorname{PoA}$ as follows.

Theorem 8 The PoA is at least $\frac{3543193}{2419209} \approx 1.46460806$.

Proof. The better lower bound is achieved by a more tricky construction, again using the one from Theorem 4. Its old items are given in $n$ optimal bins. Among these optimal bins there are $n / 4$ bins, each having a room of $1 / 3-(4 n-2) \varepsilon$ (see Observation 5). We will use this room for packing new items, and we call these bins recycled bins.

The new items will have sizes approximately $1 / 6,1 / 12,1 / 24$ and $1 / 48$. There are three subtypes from each type, these subtypes are denoted by the symbols "-", "+", and "++". The exact sizes are as follows:

$$
\begin{array}{ccrl}
1 / 6^{+}=1 / 6+19 \alpha & 1 / 6^{-}=1 / 6-20 \alpha & 1 / 6^{++}=1 / 6+999 \alpha \\
1 / 12^{+}=1 / 12+19 \beta & 1 / 12^{-}=1 / 12-20 \beta & 1 / 12^{++}=1 / 12+999 \beta \\
1 / 24^{+}=1 / 24+19 \gamma & 1 / 24^{-}=1 / 24-20 \gamma & 1 / 24^{++}=1 / 24+999 \gamma \\
1 / 48^{+}=1 / 48+19 \delta & 1 / 48^{-}=1 / 48-20 \delta & 1 / 48^{++}=1 / 48+999 \delta
\end{array}
$$

where the values of $\alpha, \beta, \gamma$, and $\delta$ are as follows: $\alpha=(4 n-2) \varepsilon, \beta=1000 \alpha$, $\gamma=1000 \beta$ and $\delta=1000 \gamma$. We ensure that $\delta$ is still very small, for example $\delta<10^{-100}$ applies.

We give the types of the recycled bins of the optimal packing. We list only the new items in these bins (any such recycled bin already contains two old medium sized items.) We will need new parameters of $a, b, c, d, e$. 
- There are $a$ bins containing one $1 / 6^{+}$and one $1 / 6^{-}$-item.

- There are $b$ bins containing one $1 / 6^{++}$-item, one $1 / 12^{+}$and one $1 / 12^{-}$item.

- There are $c$ bins containing one $1 / 6^{++}$and one $1 / 12^{++}$-item, moreover one $1 / 24^{+}$and one $1 / 24^{-}$-item.

- There are $d$ bins containing one $1 / 6^{++}$, one $1 / 12^{++}$and one $1 / 24^{++}$-item, and one $1 / 48^{+}$and one $1 / 48^{-}$-item.

- There are $e$ bins containing one $1 / 6^{++}$, one $1 / 12^{++}$, one $1 / 24^{++}$and one $1 / 48^{++}$-item.

It is easy to see that based on the definition of the sizes of the items, all such bins are valid (i.e., the items fit into the bins); in fact, all these bins are fully packed, except the last subtype where among other items the $1 / 48^{++}$-items are packed. Now we give the new bins that are created in the $N E$. For this we need some new parameters, as $x, y, z, u, t, s, v, w$.

- There are $x$ bins containing one $1 / 6^{-}$and four $1 / 6^{+}$-items, and $y$ bins containing three $1 / 6^{-}$and two $1 / 6^{++}$-items.

- There are $z$ bins containing four $1 / 12^{-}$and seven $1 / 12^{+}$-items, and $u$ bins containing nine $1 / 12^{-}$and two $1 / 12^{++}$-items.

- There are $t$ bins containing ten $1 / 24^{-}$and thirteen $1 / 24^{+}$-items, and $s$ bins containing twenty-one $1 / 24^{-}$and two $1 / 24^{++}$-items.

- There are $v$ bins containing twenty-two $1 / 48^{-}$and twenty-five $1 / 48^{+}$items, and $w$ bins containing forty-five $1 / 48^{-}$and two $1 / 48^{++}$-items.

We need to ensure that the number of items of each subtype is the same in the two (i.e., optimal and $N E$ ) packings. For this we get the next system of equations: 


$$
\begin{aligned}
& b+c+d+e=\quad 2 y \\
& \text { a }=4 x \\
& \text { a }=x+3 y \\
& c+d+e=\quad 2 u \\
& b \quad=\quad 7 z \\
& b \quad=\quad 4 z+9 u \\
& d+e=\quad 2 s \\
& c \quad 13 t \\
& \begin{array}{lll}
c & =10 t+21 s
\end{array} \\
& e=\quad 2 w \\
& d=25 v \\
& \begin{array}{ll}
d= & 22 v+45 w
\end{array}
\end{aligned}
$$

Here (1) stands for the number of $1 / 6^{++}$-items in the $O P T$ packing and in the $N E$ packing on the left-hand side and the right-hand side, respectively. Similarly, (4), (7), and (10) count the number of $1 / 12^{++}$-items, $1 / 24^{++}$-items and $1 / 48^{++}$-items, respectively.

Moreover, (2) stands for the number of $1 / 6^{+}$-items, in the $O P T$ packing and in the $N E$ packing on the left-hand side and the right-hand side, respectively. Similarly, (3) stands for the number of $1 / 6^{-}$-items. Following the list, (5) and (6) count the number of $1 / 12^{+}$and $1 / 12^{-}$-items, (8) and (9) count the number of $1 / 24^{+}$and $1 / 24^{-}$-items, and finally (11) and (12) count the number of $1 / 48^{+}$ and $1 / 48^{-}$-items.

We have 12 equations and 13 variables, the degree of freedom is one. Let us choose $w=8$, then the unique solution for the variables is as follows: $w=8$, $v=120, s=1508, t=10556, u=70122, z=210366, x=y=806403$, moreover $a=3225612, b=1472562, c=137228, d=3000, e=16$. Note that $a+b+c+d+e=4838418$ while $x+y+z+u+t+s+v+w=1905486$.

Now let us see what is the new lower bound. The number of optimal bins is $(3 / 4) n+1$. Within these bins, there are $n / 4$ recycled bins. Let us choose a new integer variable $g$, such that $n / 4=4838418 \mathrm{~g}$. Then there are $1905486 \mathrm{~g}$ new bins in the $N E$ packing, thus $O P T=(3 / 4) n+1$ while $N E^{\prime}=n+1905486 g$ (here $N E^{\prime}$ means the new value of the bins in the equilibrium). It means that by letting $g \rightarrow \infty$, our improved lower bound is

$$
P o A \geq \frac{N E^{\prime}}{O P T-1}=\frac{4 \cdot 4838418+1905486}{3 \cdot 4838418}=\frac{3543193}{2419209} \approx 1.46460806 .
$$

We still need to prove that the new packing is really a stable packing. We will call the new bins in the $N E$ as $x$-bins, $y$-bins, $\ldots, w$-bins, according to the 
number of those bins. By easy calculation we get the levels of the different types of bins. This is given in the table below. For the sake of simplicity, we use the notation $l(x)$ for the level of $x$-bins, $l(y)$ for the level of $y$-bins, and so on.

$$
\begin{array}{ll}
l(x)=5 / 6+56 \alpha & l(y)=5 / 6+1938 \alpha \\
l(z)=11 / 12+53 \beta & l(u)=11 / 12+1818 \beta \\
l(t)=23 / 24+47 \gamma & l(s)=23 / 24+1578 \gamma \\
l(v)=47 / 48+35 \delta & l(w)=47 / 48+1098 \delta
\end{array}
$$

In what follows, we show that no item moves in the $N E$ packing. A $k$-bin means a bin with exactly $k$ items, and a $k^{+}$-bin means a bin with at least $k$ items. We illustrate the principle with the 5 -bins (i.e., the $x$-bins and $y$-bins) and items therein.

A $1 / 6^{+}$-item or a $1 / 6^{-}$-item does not want to go to a 2 -bin, since there the level of the bin is $2 / 3+n \varepsilon<2 / 3+\alpha$ (see Observation 5), and the size of an $x$-item is at most $1 / 6+19 \alpha$, thus the increased level of the target bin would be at most $5 / 6+20 \alpha$, which is smaller than $l(x)$. A $1 / 6^{++}$-item would create a higher level, but still lower than $l(y)$, therefore an item of that size does not want to move either. A 1/6-type item does not fit into any other 5-bin, neither into any $11^{+}$-bin. We conclude that no $1 / 6$-type item moves.

In a very similar way, which is a matter of routine to check, it can be verified that the other items do not move either. Details are left to the reader.

Notes. We do not apply any big item in our construction. The reason is that the weight of a big item itself is 1 , and the weight of any bin not containing a big item is at most 1 . It follows that if there is at least one bin containing a big item, any small item wants to move there. As a consequence, the bin of any big item will be almost completely full in any $N E$ packing (more exactly, if $x$ is the size of the smallest item being packed into a bin without a big item, then the level of any bin with a big item is bigger than $1-x$ ). Hence, it seems that applying big items in a construction is not advantageous if we want to get a large $N E / O P T$ ratio in the considered setting.

We also note that our construction applies to items of sizes around $1 / 6$, $1 / 12,1 / 24,1 / 48$. If we go further and include also items of sizes around $1 / 96$ (or even smaller ones) we will get certain improvement on the lower bound, but this improvement is really very small. If the smallest items were about $1 / 24$ (instead of about 1/48), the implied lower bound would be about 1.464599. 


\subsection{An Upper Bound of $3 / 2$ for $S 1$}

Here we prove the following bound.

Theorem 9 For setting $S 1$ the PoA is at most 3/2.

Proof. Let us consider an arbitrary $N E$ packing. Recall that an item is called big if its size is above $1 / 2$ otherwise it is small. We define the following two bin types:

B1: The bin contains one big item, and possibly several further (small) items.

B2: The bin contains only small items.

Since all big items have weight 1 , the weight of any B1-bin is at least 1, while the weight of any B2-bin is at most 1 . It follows that any item of a B2-bin has the intention to move into a B1-bin. Since the considered packing is a $N E$, no item of a B2-bin fits into a B1-bin.

If there is no B2-bin, then every $N E$ bin contains a big item, thus the packing is optimal. If there is only one B2-bin, still $N E \leq O P T+1$ holds. Thus, let us suppose that there exist at least two B2-bins. Consider the B2-bin with the smallest level — let $l_{1}$ denote this level — and let $X$ be the smallest item in this bin. Now consider the B2-bin with the second smallest level - let $l_{2}$ denote this level, then $l_{2} \geq l_{1}$ - and let $Y$ be the smallest item in this bin.

We distinguish two cases.

Case 1: $l_{2}>2 / 3$.

It means that the level of any B2-bin is above $2 / 3$, except possibly the bin containing $X$. If also the level of any B1-bin is above $2 / 3$, we are done by an averaging argument. Thus let us suppose that there is a B1-bin with smaller level. Since no item of a B2-bin fits into a B1-bin, it follows that any small item in the B2-bins has size above 1/3. Let us denote the number of B1 and B2-bins by $x$ and $y$, respectively. Obviously, $O P T \geq x$.

If $y \leq x / 2$, we have

$$
N E=x+y \leq \frac{3}{2} x \leq(3 / 2) O P T
$$

Suppose now that $y>x / 2$. Every B2-bin contains exactly two items, both are larger than 1/3, and any B1-bin contains a big item. Two big items cannot be packed into a common bin, and a big item can share a bin with at most one

small item which is larger than $1 / 3$. Thus $O P T \geq x+(2 y-x) / 2=x / 2+y$. Hence,

$$
\frac{N E}{O P T} \leq \frac{x+y}{x / 2+y}=1+\frac{x / 2}{x / 2+y} \leq 1+\frac{x / 2}{x / 2+x / 2}=3 / 2
$$


Case 2: $l_{1} \leq l_{2} \leq 2 / 3$.

Under this assumption it follows that $X>1 / 3$, as $X$ would like to go into the bin of $Y$, but cannot move, since $X$ does not fit into the bin of $Y$.

Assume $Y \leq 1 / 3$. Since $Y$ does not fit into any other bin apart from the bin of $X$, the level of any bin apart from the bins of $X$ and $Y$ is above $2 / 3$. Thus, $N E \leq(3 / 2) O P T+2$ and we are done.

Therefore, also $Y>1 / 3$ holds. If there are at least two items in the bin of $X$, then $X \leq 1 / 3$ would follow, also if there were at least two items in the bin of $Y$. We conclude that both $X$ and $Y$ are the only items in their bins. This is a contradiction, since both are small items. They rather share their bins than stay alone. This completes the proof of the theorem.

\section{Three Levels of Weights: Setting $S 2$}

We found quite close bounds in the considered simple setting $S 1$. In this section we consider another, more complicated setting, denoted as $S 2$, where the $P o A$ is a bit smaller. For this, we give larger weights to certain smaller items as well, but then it is necessary to make a more detailed analysis.

We choose the item weights as follows. Let the items be denoted as big, medium, small, and tiny if their sizes are larger than $1 / 2$, larger than $1 / 3$ and at most $1 / 2$, larger than $1 / 4$ and at most $1 / 3$, and finally at most $1 / 4$, respectively. The classes and their weights are described in Table 2 . In the relation between size and weight, 'small' and 'tiny' behave in the same way, but their distinction will play a role in the analysis of $P o A$.

\begin{tabular}{|r|c|c|}
\hline Class & Size $s_{i}$ & Weight $g_{i}$ \\
\hline big & $1 / 2<s_{i}$ & $g_{i}=1$ \\
\hline medium & $1 / 3<s_{i} \leq 1 / 2$ & $g_{i}=1 / 2$ \\
\hline small & $1 / 4<s_{i} \leq 1 / 3$ & $g_{i}=s_{i}$ \\
\hline tiny & $s_{i} \leq 1 / 4$ & $g_{i}=s_{i}$ \\
\hline
\end{tabular}

Table 2: Weights for setting $S 2$ 


\subsection{A Lower Bound for $S 2$}

Here we give a lower bound for the PoA as

$$
P o A \geq \frac{4}{3}+\frac{1}{11}+\frac{1}{3} \cdot \sum_{k=3}^{\infty} \frac{1}{3 \cdot 2^{k}-1} \approx 1.4528
$$

Theorem 10 For setting $S 2$ the PoA is at least 1.4528.

Proof. Consider the construction given in Theorem 7 where we gave a packing for instance $I^{\prime}$ which was a $N E$ for setting $S 1$. This packing is not a $N E$ for $S 2$ since any bin containing a medium item has level close to $2 / 3$ and weight close to $5 / 6$. Thus, we would get an improving step by moving an item of size $a_{1}=1 / 6+n \varepsilon$ into that bin.

Hence, we construct a new instance $I^{\prime \prime}$ where each item of size $a_{1}$ is replaced by two items of size $a_{2}=1 / 12+n \varepsilon$ and the other items of $I^{\prime}$ are left unchanged.

Again, there is an optimal packing using only $(3 / 4) n+1$ bins. We use the same packing strategy for the items in $I^{\prime \prime}$ as for the items in $I^{\prime}$.

Now in the $N E$ packing the $a_{1}$ items are missing, but there are three times more bins of type $B_{2}$ containing eleven $a_{2}$ items. The number of newly created bins is therefore

$$
\frac{n}{4} \cdot\left(\frac{3}{3 \cdot 2^{2}-1}+\sum_{i=3}^{k} \frac{1}{3 \cdot 2^{k}-1}\right) .
$$

The total number of bins in the packing is

$$
n+\frac{n}{4} \cdot\left(\frac{3}{3 \cdot 2^{2}-1}+\sum_{i=3}^{k} \frac{1}{3 \cdot 2^{k}-1}\right) .
$$

Thus, the lower bound follows, once we show that this packing is a $N E$. We show that no item can make an improving step. Now the difference is only regarding the option that a new item perhaps wants (and can) move into a bin of a medium item. It is sufficient to see that an item $a_{2}$ does not want to move into a bin of a medium item. In such a target bin the total weight is maximal if the biggest small item is packed together with a medium item. Hence the maximum weight is bounded by $1 / 2+S_{n}=1 / 2+1 / 3-(n / 2) \varepsilon=5 / 6-(n / 2) \varepsilon$. The weight of a current bin of an $a_{2}$ item is $11 \cdot(1 / 12+n \varepsilon)$. Should the $a_{2}$-item move, the weight in the target bin would become $5 / 6-(n / 2) \varepsilon+1 / 12+n \varepsilon=11 / 12+(n / 2) \varepsilon$, which is smaller than the weight of the current $a_{2}$-bin. Hence, this packing gives a $N E$.

Let us remark that in the previous construction there are no big items as their bins would be filled too much. We have medium items, and considerably smaller items which are close to $1 / 12$, and even smaller items. It means that 
if we want to define a "better" weighting system which makes the PoA (much) smaller, it does not help if we give larger weight for the items between $1 / 3$ and $1 / 12+\delta$ for some $\delta>0$.

\subsection{An Upper Bound of $16 / 11 \approx 1.4545$ for $S 2$}

In this section we prove that the $P o A$ of our game is at most $16 / 11 \approx 1.4545$. For this, we have to make a much more detailed classification of the bins in a $N E$.

\subsubsection{Classification of Bin Types and Some Simple Observations}

In this subsection, we will classify the bins in a NE with respect to the corresponding items. This is accompanied by some simple, but useful observations.

Let us consider an arbitrary $N E$ packing. We define the following basic bin types:

B The bin contains one big item, and possibly several further items.

MM The bin contains two medium items, and possibly several further (i.e., small or tiny) items.

M The bin contains exactly one medium item, no big item, and possibly several further (i.e., small or tiny) items.

ST The bin contains only small or tiny items.

Some of the bin types will be further divided later. First, we make several simple observations which hold for a $N E$.

The weight of any B-bin or MM-bin is at least 1 , and the weight of any ST-bin is at most 1. It follows that any item of a ST-bin would like to move into a B-bin or into a MM-bin. We get our first observation.

Observation 11 No item of a ST-bin fits into a B-bin or into a MM-bin in a $N E$.

A medium item has an intention to go into a B-bin. The reason is that the increased weight in the target B-bin would be at least $3 / 2$, while the weight of a MM-bin is smaller than $2 \cdot 1 / 2+1 / 3=4 / 3$, and the weight of a M-bin is smaller than $1 / 2+2 / 3=7 / 6$. This results in the next observation.

Observation 12 No medium item of a MM-bin or M-bin fits into a B-bin in a NE. 
Any small item and any tiny item with size above $1 / 6$ in an M-bin has an intention to go into a B-bin. The reason is that the increased weight in the target B-bin would be at least $1+1 / 6$, and the weight of an M-bin is smaller than $1 / 2+2 / 3=7 / 6$. We conclude the following observation.

Observation 13 No small item and no tiny item of size above 1/6 in a M-bin fits into a B-bin in a NE.

Now we distinguish the bin types more detailed and divide them into several subtypes. Any subtype will have certain properties. To make this possible, we will use irregular bins, which possibly do not satisfy some of the required properties. These irregular bins are excluded from later consideration. At any time when we encounter an irregular bin, the number $C$ of irregular bins is increased by one.

\section{Type B}

Type B is divided into the following four subtypes:

B0 The bin contains a big item, and no other item. The big item in this kind of bin is also called superbig.

BTf The bin contains a big item, at least one tiny item, and possibly several further items.

BM The bin contains a big item and a medium item and no further item.

BS The bin contains a big item and a small item and no further item.

Note that any B-bin falls into exactly one subtype. Moreover the level of a BM-bin is above $5 / 6$, and the level of a BS-bin is above $3 / 4$.

Now we focus on the BTf-bins, if there are any. Let us consider the bin among the BTf-bins with smallest weight. Consider a tiny item in this bin. This item has an intention to go into any other BTf-bin. It cannot move, it means that the level of any other BTf-bin is above 3/4. The BTf-bin with smallest level is considered as an irregular bin. That means, we now have $C=1$, and any regular BTf-bin has a level above $3 / 4$.

\section{Type MM}

The MM type is not divided into subtypes.

\section{Type M}

There can be at most one M-bin with a single medium item in a $N E$. We exclude this bin from consideration, hence $C=2$. Among the remaining M-bins, we define the following subtypes: 
MTf The bin contains among other items at least one tiny item.

MS1 The bin contains one medium item and one small item and no other item.

MS2 The bin contains one medium item and two small items and no other item.

Note that the classification is complete, i.e., any M-bin belongs to one of the subtypes MTf, MS1, MS2.

Among the MTf-bins (if there are any), consider the bin with smallest weight. The tiny item in this bin would like to move into any other MTf-bin but cannot in a $N E$. The MTf-bin with smallest weight is irregular and we set $C=3$. All regular MTf-bins have level greater than 3/4.

The medium and the small item of a MS1-bin will be called supermedium and supersmall, respectively. Any small item in a MS1-bin has the intention to move to any other MS1-bin. Since it cannot move in a $N E$, it means that the level of any MS1-bin is above $2 / 3$. This argument works whenever the number of MS1-bins is different from 1. For the case when there is just one MS1 bin, we exclude this, and from now $C=4$.

\section{Type ST}

Let us consider the ST-bins which do not have any tiny item. Any such bin contains several small items only. Since any three small items fit into a common bin (and the total size of three small items is larger than $3 / 4$ and the total size of at most two small items is at most $2 / 3$ ), there can be at most one ST-bin with at most two small items. If there is such a bin, it is considered as irregular, hence $C=5$. All other ST-bins without tiny items contain exactly three small items. We obtain a partition into the following subtypes:

Tf The ST-bin contains at least one tiny item.

S3 The ST-bin contains exactly three small items.

This is again a complete classification, as all regular ST-bins are either Tfbins or S3-bins.

Consider the Tf-bin with smallest level. A tiny item in this bin has an intention to go into any other Tf-bin. Since it cannot move in a $N E$, the level of any other Tf-bin is above $3 / 4$. The Tf-bin with smallest level is considered as an irregular bin, hence $C=6$.

We conclude that the level of any regular ST-bin is above 3/4. Small items in a S3-bin are also called distinguished small items. All special items which have been introduced for the different bin types are listed in Table 3.

During the classification of the bins we get strict lower bounds for the levels of all regular bins in any $N E$, i.e., the levels of bins of that type are higher than the given value. These lower bounds, denoted as "minimum level", are summarized in Table 4 with respect to the corresponding bin type. 


\begin{tabular}{|l|l|}
\hline Name of special item & \multicolumn{1}{c|}{ Definition } \\
\hline superbig & big item in B0-bin \\
\hline supermedium & medium item in MS1-bin \\
\hline supersmall & small item in MS1-bin \\
\hline distinguished small & small item in S3-bin \\
\hline
\end{tabular}

Table 3: Special items of the bin types

\begin{tabular}{|r|c|c|c|c|c|}
\hline Bin type & B0 & BTf & BM & BS & MM \\
\hline Minimum level & $1 / 2$ & $3 / 4$ & $5 / 6$ & $3 / 4$ & $2 / 3$ \\
\hline \hline Bin type & MTf & MS1 & MS2 & Tf & S3 \\
\hline Minimum level & $3 / 4$ & $2 / 3$ & $5 / 6$ & $3 / 4$ & $3 / 4$ \\
\hline
\end{tabular}

Table 4: Minimum level of regular bins

\subsubsection{Proof of the Upper Bound}

The proof of our upper bound of $16 / 11$ is based on case distinction. In this case distinction we will introduce an additional weight for the items. This new weight will be denoted by $v$, and will be called the value. The value $v(a)$ of any item $a$ consists of the sum of two parts. One part is the scaled size $c(a)$, another part is the bonus $b(a)$. Both the scaled size and the bonus are nonnegative. Formally, the value of an item $a$ is

$$
v(a)=c(a)+b(a) .
$$

This type of weight was efficiently applied in [7].

We assign the values to the items so that the total value of the items in any regular $N E$ bin will be at least one. We will also show that the total value of any bin (i.e., any set of items with total size at most 1 ) is at most 16/11. If this holds, then the claimed upper bound follows by the following lemma.

Lemma 14 Suppose that the value of any regular $N E$ bin is at least 1 , there are at most $C$ irregular bins, and the value of any optimal bin is at most 16/11. Then, $N E \leq \frac{16}{11} \cdot O P T+C$.

Proof. Let the total value of the items be denoted by $W$. Then $N E-C \leq$ $W \leq \frac{16}{11} \cdot O P T$. 
Now we distinguish several cases.

Case 1. There exists a B0-bin with level below $2 / 3$.

Obviously, the space left in the B0-bin with smallest level is larger than $1 / 3$, i.e., any small item fits into this room. Thus, by Observations 11, 12, and 13 we can exclude the existence of bins which contain tiny or small items and these items have an intention to move into the B-bin. In particular, we can exclude the existence of any ST-bin, any MS1-bin, and any MS2-bin. We consider two subcases.

Case 1.1. The level of every B0-bin is at least $7 / 12$.

Set $c(a)=12 / 11 \cdot s(a)$. The medium items of MM and MTf-bins get 2/11 bonus, the big items that are not superbig get also 2/11 bonus, and the superbig items get $4 / 11$ bonus. (The other, not mentioned items do not have positive bonus.) The total scaled size of any bin is at most $12 / 11$. Thus, in order to show that the value of any optimal bin is at most $16 / 11$, we have to guarantee that the total bonus of any bin is at most $4 / 11$. If the bin contains a superbig item, this holds, as no other big item fits into the bin, moreover no medium item of some MM or MTf-bin fits into the bin by Observation 12. Otherwise, the bin contains at most two items with positive bonus, and the total bonus is at most $2 \cdot 2 / 11$.

Now we show that the value of any regular bin in a $N E$ is at least 1 . The value of any of the MTf, BM, BTf, BS-bins is at least $12 / 11 \cdot 3 / 4+2 / 11=1$, since the level of any such bin is at least $3 / 4$, and the bin has an item with positive bonus. The value of a B0-bin is at least $12 / 11 \cdot 7 / 12+4 / 11=1$. Finally, the level of any MM-bin is above $2 / 3$, thus the value of such a bin is at least $12 / 11 \cdot 2 / 3+4 / 11=12 / 11>1$.

Case 1.2. There exists a B0-bin with level below $7 / 12$.

Now the available space in the B0-bin with smallest level is larger than 5/12. It follows that any medium item in a MM or MTf-bin is larger than 5/12. Recall that neither MS1 nor MS2-bins exist.

We define the values for this case. Set $c(a)=10 / 11 \cdot s(a)$. The medium items of MM and MTf-bins get 2/11 bonus, the big items that are not superbig get $4 / 11$ bonus, and the superbig items get $6 / 11$ bonus.

We show that the value of any optimal bin is at most $16 / 11$ by ensuring that the total bonus of any optimal bin is at most $6 / 11$. If the bin contains a superbig item, this holds, as no other big item fits into the bin, and no medium item with positive bonus fits into the bin by Observation 12. Suppose the bin contains a big item that is not superbig. Obviously, the bin cannot contain another big item and it can contain at most one medium item with bonus 2/11. Finally, if the bin contains no big item, it can contain at most two medium items with total bonus $4 / 11$. 
Now let us compute the values of the $N E$ bins. The value of a B0-bin is at least $1 / 2 \cdot \frac{10}{11}+6 / 11=1$. The value of any of the BM, BTf, BS-bins is at least $10 / 11 \cdot 3 / 4+4 / 11=23 / 22>1$, since the level of any such bin is at least $3 / 4$, and the big item has bonus $4 / 11$. The value of a MM-bin is at least $10 / 12 \cdot \frac{10}{11}+2 \cdot 2 / 11=\frac{37}{33}$.

Since no MS1, MS2, ST-bins exist, it remains to examine the MTf-bins. If the level of a MTf-bin is at least 9/10, the value of the bin is at least 9/10 . $10 / 11+2 / 11=1$. If there is only one MTf-bin with level below $9 / 10$, we exclude this bin by calling it irregular, $C$ is increased by one to $C=7$ and we are done again. Let us suppose there are at least two such bins and consider the bin $\beta$ with smallest weight. The bin $\beta$ contains a tiny item $\tau$ since it is a MTf-bin. Tiny item $\tau$ has an intention to go into another MTf-bin with level below 9/10, but cannot move, which means that the size of $\tau$ is larger than $1 / 10$. Hence, $\tau$ would fit into the B0-bin with level below $7 / 12$ and the target B0-bin would get a weight larger than $1+1 / 10=11 / 10$. Bin $\beta$ consists of a medium item with weight equal to $1 / 2$ plus further items of total size at most $2 / 3$. Thus, the weight of $\beta$ without $\tau$ is at most $1 / 2+2 / 3-1 / 10=16 / 15<11 / 10$. Since we considered a $N E$, we get a contradiction. Consequently, any regular $N E$ bin has value at least 1 . This finishes Case 1 .

After the investigation of Case 1, we suppose in the following that the level of any B0-bin is at least $2 / 3$.

Case 2. The level of any MS1-bin is at least $3 / 4$.

For any item $a$ let the scaled size be $c(a)=4 / 3 \cdot s(a)$. Note that bins have value at least 1 if the level of the bin is at least $3 / 4$. So we assign a positive bonus only to those $N E$ bins where the level is possibly below $3 / 4$. This concerns the following two bin types:

- B0-bins. The superbig items get 4/33 bonus.

- MM-bins. Any medium item in these bins gets 2/33 bonus.

First we have to show that the total value of any (optimal) bin is at most 16/11. The total scaled size of the items in a bin is at most $4 / 3$. Thus it suffices to show that the total bonus of items in any bin is at most $16 / 11-4 / 3=4 / 33$. Three medium or big items cannot be in the same bin. If there is only one item with positive bonus in a bin, this bonus is at most $4 / 33$ and we are done. If there are two items with positive bonus and both are medium items, we are again done. Finally suppose that there is one superbig item in the bin, and one medium item from some MM-bin. This is a contradiction by Observation 12 .

Now we bound the value of the $N E$ bins. It is clear that the value of any regular $N E$ bin is at least 1, except possibly MM-bins and B0-bins. But for 
MM-bins the value is at least $4 / 3 \cdot 2 / 3+2 \cdot 2 / 33=100 / 99>1$. Analogously, for B0-bins the value is at least $4 / 3 \cdot 2 / 3+4 / 33=100 / 99>1$.

In the following we suppose that the level of any B0-bin is at least $2 / 3$ and there is a MS1-bin with level below 3/4.

Next, we prove two lemmas which are required for the further case distinction.

Lemma 15 Apart from at most one irregular bin the level of any MTf-bin in a $N E$ is larger than $19 / 24$.

Proof. Let us focus on the MTf-bins with level at most 19/24; denote this subtype by MT2. Any tiny item in such a bin would like to move into another MT2-bin with larger or the same weight, but cannot. It means that any tiny item has size above 5/24 in any MT2-bin apart from at most one irregular bin. Exclude this irregular bin.

Now let us focus on the remaining MT2-bins. Any such bin has level at most $19 / 24$, and any tiny item in the bin has size above $5 / 24$. If the bin contains one medium item, a small item and also a tiny item, the level of the bin is above $1 / 3+1 / 4+5 / 24=19 / 24$, a contradiction. Thus the bin contains only one medium item and several tiny items. The bin cannot contain three tiny items, because the level would be above $1 / 3+3 \cdot 5 / 24=23 / 24$. Analogously, the bin cannot contain only one tiny item, because the level would be at most $1 / 2+1 / 4=3 / 4$, although we have previously seen that any MTf-bin has level above $3 / 4$. Thus we conclude that a regular MT2-bin contains a medium item, and exactly two tiny items.

The weight of any regular MT2-bin is at most $1 / 2+19 / 24-1 / 3=23 / 24$ (the weight of the medium item plus the maximum total size for the two tiny items in the bin). Recall that there is a MS1-bin with level at most 3/4. If a tiny item moves into that MS1-bin, the total weight in the target MS1-bin is above $1 / 2+1 / 4+5 / 24=23 / 24$. It means that there is at most one MT2-bin which has been counted as irregular.

According to lemma, $C$ is possibly increased by one, so we have now $C=8$. Moreover we know that any regular MTf-bin has level above 19/24. In the following we will use that there are at least two MS1 bins. If this is not the case (there is at most one MS1 bin), we consider this bin as irregular, so from now $C=9$.

Lemma 16 (i) No supersmall item and no distinguished small item fits into another MS1-bin.

(ii) Let $M_{1}$ and $M_{2}$ be two different supermedium items and let $S$ be a supersmall or distinguished small item. Then $M_{1}+M_{2}+S>1$. 
(iii) Let $M_{1}$ be a supermedium item and let $S$ be a supersmall or distinguished small item. Moreover, let $M$ be a medium item (which is not necessarily supermedium). Then, $M_{1}+M+S>1$.

Proof. Recall that the supermedium and supersmall items are packed into MS1-bins, and distinguished small items are packed into S3-bins.

(i) The weight of a MS1-bin is at most $1 / 2+1 / 3=5 / 6$, as the weight of a medium item is $1 / 2$, and the weight of a small item is at most $1 / 3$. Similarly, the weight of a S3-bin is at most 1 . On the other hand the weight of the target MS1-bin is larger than $1 / 2+2 \cdot 1 / 4=1$ if the moving small item fits there.

(ii) No matter whether $S$ is a supersmall or distinguished small item, $S$ cannot be in the same bin together with both supermedium items. Suppose without loss of generality that $S$ is not in the same bin together with $M_{1}$. Let $S_{1}$ be the supersmall item in the MS1-bin of $M_{1}$. Note that $M_{2}>S_{1}$ because any medium item is larger than any small item. Then $M_{1}+M_{2}+S>M_{1}+S_{1}+S>1$, where the second inequality follows by (i).

(iii) If $S$ is a supermedium item in the same bin as $M_{1}$, then no supersmall item from some other MS1-bin fits here by (i). Since $M$ is larger than a supersmall item, the claim holds. Now suppose that $S_{1}$ is the supersmall item in the MS1-bin of $M_{1}$, and $S$ is different from $S_{1}$. We get again $M_{1}+M+S>$ $M_{1}+S_{1}+S>1$. The first inequality holds as a medium item is larger than a small item and the second inequality follows again by (i).

Now we continue with our main case distinction. We will distinguish between the existence or non-existence of Tf-bins.

Case 3. There is no Tf-bin.

We introduce the following values for the items. Let $c(a)=12 / 11 \cdot s(a)$. The superbig items get $3 / 11$ bonus, the further big items that are not superbig get $2 / 11$ bonus. The supermedium items get $2 / 11$ bonus. The medium items of MM, MTf and MS2-bins get 3/22 bonus. Finally, the supersmall items and distinguished small items get $1 / 11$ bonus.

To show that the value of any optimal bin is at most $16 / 11$, it suffices to see that the total bonus of any bin is at most $4 / 11$. Consider a bin with a superbig item. Of course, no other big item fits into the bin. Moreover, no medium item of some MM or M-bin fits into the bin, by Observation 12. If there is a further item with positive bonus together with the superbig item, it is a supersmall or distinguished small item. But there can be at most one small item in this bin, and it has bonus at most $1 / 11$. We are done in this case.

Suppose there is a big item in the bin which is not superbig. There can be at most one further item in the bin (which is not big) with positive bonus, and the total bonus is at most two times 2/11. Suppose next that there are two medium items in the bin (three or more is not possible). If at least one of them 
is supermedium, then there is no more item in the bin with positive bonus by Lemma 16(iii). If both medium items are medium but not supermedium, there can be at most one further item in the bin with positive bonus. This must be a supersmall or distinguished small item. Hence, the total bonus is at most $2 \cdot 3 / 22+1 / 11=4 / 11$. Finally, if there is at most one medium item in the bin, there can be at most two further items in the bin each having at most $1 / 11$ bonus. Hence, the total bonus is at most $2 / 11+2 \cdot 1 / 11$, and we are done.

Now we show that the value of any regular $N E$ bin is at least 1 . The value of a B0-bin is at least $12 / 11 \cdot 2 / 3+3 / 11=1$. The value of any of the BTf, $\mathrm{BM}$, BS-bins is at least $12 / 11 \cdot 3 / 4+2 / 11=1$, since the level of any such bin is at least $3 / 4$, and the bin has an item with $2 / 11$ bonus. The weight of a MM-bin is at least $12 / 11 \cdot 2 / 3+2 \cdot 3 / 22=1$. The weight of a S3-bin is at least $12 / 11 \cdot 3 / 4+3 \cdot 1 / 11=12 / 11>1$. The weight of a MS1-bin is at least $12 / 11 \cdot 2 / 3+2 / 11+1 / 11=1$. The weight of a MS2-bin is at least $12 / 11 \cdot 5 / 6+3 / 22=23 / 22>1$. Finally by Lemma 15 the weight of a MTf-bin is at least $12 / 11 \cdot 19 / 24+3 / 22=1$, since there is a medium item with $3 / 22$ bonus in the bin.

Now we are at the last case to be considered. Recall that the level of any B0-bin is at least 2/3, and there is a MS1-bin with level below 3/4.

Case 4. There exists at least one Tf-bin.

In this final case the analysis is more difficult. Recall that any small item of a MS1-bin has an intention to go into another MS1-bin but cannot fit. Let the level of the MS1-bin with smallest level be denoted by $\ell$, where $2 / 3<\ell<3 / 4$. We consider this bin as irregular, increasing $C$ to $C=10$. If there is no other MS1bin, we are done with this subcase, as it may be treated like Case 2. Otherwise, we conclude that the level of any regular MS1-bin is at least $\ell$, and any small item in that MS1-bin is larger than $1-\ell$. For convenience, let $\ell=2 / 3+2 x$ with $0<x<1 / 24$. Consequently, the size of any small item in a regular MS1-bin is above $1-(2 / 3+2 x)=1 / 3-2 x$. Thus, the weight of any regular MS1-bin is above $1 / 2+1 / 3-2 x=5 / 6-2 x$.

Let us now consider the Tf-bins. Suppose there are two Tf-bins with level at most $11 / 12-x$. Consider the bin with smallest weight among these two bins. Any item in this bin has the intention to move to the other Tf-bin. It follows that any such item has size above $1 / 12+x$.

But such an item has also an intention to move into the MS1-bin with level below 3/4: It fits there as there is at least $1 / 4$ idle space in the target bin. Moreover, the increased weight of the target bin will be more than $(5 / 6-2 x)+$ $(1 / 12+x)=11 / 12-x$. This is a contradiction since the weight of the bin of the considered item is at most $11 / 12-x$.

It follows that the level of any Tf-bin is above $11 / 12-x$, with at most one 
irregular bin. We exclude this bin, and we have now $C=11$. Let the tiny item of an arbitrary (but fixed) regular Tf-bin be denoted by $X$.

We will assign now values to the items. The coefficient of the scaled size is the reciprocal of $11 / 12-x$, i.e.,

$$
c(a)=\frac{12}{11-12 x} \cdot s(a)
$$

for any item $a$.

Let us consider the other bin types.

(i) B-bins: The big item in a B-bin gets bonus $\frac{2-12 x}{11-12 x}$. Since the tiny item $X$ in a regular Tf-bin has an intention to move into a B-bin but cannot move, it follows that the level of any B-bin is above $3 / 4$. Thus, the total value of a B-bin is at least

$$
3 / 4 \cdot \frac{12}{11-12 x}+\frac{2-12 x}{11-12 x}=1 .
$$

(ii) MM-bins: The level of any such bin is at least 3/4, since otherwise $X$ would move here. Any medium item in the MM-bins gets bonus $\frac{1-6 x}{11-12 x}$. Thus the value of the MM-bin is at least

$$
3 / 4 \cdot \frac{12}{11-12 x}+2 \cdot \frac{1-6 x}{11-12 x}=1 .
$$

(iii) M-bins: We know that the level of any regular MTf-bin is above 19/24. We give $\frac{3-24 x}{22-24 x}$ bonus to the medium item of the MTf-bin. Then the value of any such bin is at least

$$
19 / 24 \cdot \frac{12}{11-12 x}+\frac{3-24 x}{22-24 x}=1 .
$$

Consider the MS1-bins. In any such bin there is a supermedium item, and a supersmall item. The supermedium item gets bonus $\frac{2-12 x}{11-12 x}$ and the supersmall item gets bonus $\frac{1-6 x}{11-12 x}$. Recall that the level of a regular MS1-bin is at least $\ell=2 / 3+2 x$. Then the value of such a bin is at least

$$
(2 / 3+2 x) \cdot \frac{12}{11-12 x}+\frac{2-12 x}{11-12 x}+\frac{1-6 x}{11-12 x}=\frac{11+6 x}{11-12 x} \geq 1 .
$$

In any MS2-bin there is one medium item and two small items, and the level of the bin is at least $5 / 6$. The medium item gets bonus $\frac{1-6 x}{11-12 x}$. Then the total value of the bin is at least

$$
5 / 6 \cdot \frac{12}{11-12 x}+\frac{1-6 x}{11-12 x}=\frac{11-6 x}{11-12 x} \geq 1 .
$$

(iv) ST-bins: By definition, the value of any Tf-bin is at least 1 without bonus. As a final case, we give bonus for the items in the S3-bins. The level of 
any such bin is above $3 / 4$, and the bin contains three distinguished small items. Any distinguished small item gets bonus $\frac{1-6 x}{11-12 x}$. The value of the bin is at least

$$
3 / 4 \cdot \frac{12}{11-12 x}+3 \cdot \frac{1-6 x}{11-12 x}=\frac{12-18 x}{11-12 x}>\frac{11-12 x}{11-12 x}=1,
$$

since $0<x<1 / 24$. We conclude that any regular $N E$ bin has value at least 1 .

We show now that any optimal bin has value at most $16 / 11$. The scaled size of the items in the bin is at most $\frac{12}{11-12 x}$. Thus we have only to prove that the total bonus of the items is at most $\frac{16}{11}-\frac{12}{11-12 x}=\frac{44-192 x}{121-132 x}$. Instead of this, we show that the total bonus is at most $\frac{4-24 x}{11-12 x}$ in any bin. This is sufficient, as

$$
\frac{44-192 x}{121-132 x}-\frac{4-24 x}{11-12 x}=\frac{72 x}{121-132 x} \geq 0 \text {. }
$$

(i) There is a big item in the bin: In this case the bin can contain at most one further item with positive bonus. Hence, the total bonus is at most $2 \cdot \frac{2-12 x}{11-12 x}=$ $\frac{4-24 x}{11-12 x}$.

(ii) There are two medium items in the bin: The biggest bonus among the medium items belongs to the supermedium items, and this bonus is $\frac{2-12 x}{11-12 x}$. If no further item has bonus in the bin, we are done.

Suppose there are some other items in the bin with positive bonus. That can be at most one small item as otherwise the level would be greater than one. Assume that there is one supersmall or a distinguished small item in the bin, together with the two medium items. In this case, by Lemma 16(iii), both medium items are simple medium items, i.e., none of them is supermedium. Since $\frac{3-24 x}{22-24 x}$ is the largest bonus among the simple medium items, the total bonus is at most

$$
2 \cdot \frac{3-24 x}{22-24 x}+\frac{1-6 x}{11-12 x}=\frac{4-30 x}{11-12 x} \leq \frac{4-24 x}{11-12 x} .
$$

(iii) There is one medium item in the bin: Then, there can be at most two more items in the bin with positive bonus. In this case the total bonus is at most

$$
\frac{2-12 x}{11-12 x}+2 \cdot \frac{1-6 x}{11-12 x}=\frac{4-24 x}{11-12 x} .
$$

(iv) There is no medium item in the bin: Then there are at most three small items in the bin with total bonus at most

$$
3 \cdot \frac{1-6 x}{11-12 x}=\frac{3-18 x}{11-12 x} \leq \frac{4-24 x}{11-12 x} .
$$

This completes the analysis of the final Case 4.

The main result of Section 4 is summarized in the following theorem.

Theorem 17 The PoA of the game in setting S2 is at most 16/11. 


\section{Conclusions}

We have investigated the price of anarchy in selfish bin packing games under two types of weight assignments. In the setting of weights which is more sensitive to item size, the $P o A$ is proven to be between 1.4528 and $16 / 11 \approx 1.4545$. The lower bound is definitely not tight, as it can slightly be improved by a more complicated construction. Also, it is possible that the upper bound could be decreased a bit. It is very likely, however, that the exact determination of the PoA will require a rather complicated argument.

Parametric version. The construction in the proof of our Theorem 8 does not apply any items larger than $1 / 2$. In this way it also yields a modest improvement for the parametric version of size-proportional cost sharing in the case of $t=2$ (i.e., where every item size is at most $1 / t=1 / 2$ ). The previously best lower bound of 1.464571 on the $P o A$ is due to Epstein et al. [10], which is now improved to a value slightly larger than 1.464608 .

Beyond $S 1$ and $S 2$. Another option would be to introduce a refinement of setting $S 2$. We follow the harmonic sequence, define weight 1 for items having sizes above $1 / 2$, weight $1 / 2$ for items having sizes at most $1 / 2$ and bigger than $1 / 3$, weight $1 / 3$ for items having sizes above $1 / 4$ but at most $1 / 3$, and the weight of any smaller item equals the size of the item. In this setting called $S 3$, perhaps the PoA is smaller, but even if this is true, the investigation seems hard. An analogous sequence of settings $S 4, S 5, \ldots$ can be defined in the obvious way; nevertheless it is not at all clear whether the PoA of these models decreases as the index gets large.

Restrictions on selfish moves. In connection with the processes of selfish moves, one can make further refinements inside the weighting classes by imposing restrictions on the moves. We mention two such possibilities:

- there is a complete linear order (or only a partial order) on the set of items, indicating their relative importance, and an item $i$ is allowed to move only if no item more important than $i$ can make a selfish move;

- every item is allowed to move, but if an item moves, it must move in the most advantageous way, i.e., into a bin in which it minimizes its current cost.

The effect of these assumptions on the PoA, and probably also of some further ones, would be worth investigating. 
Flexible weights. Finally, we may consider weights which may depend on the packing. In this model an input of items is given, and the items are already packed. Depending on the current packing we may enforce the items to move, by giving appropriate weights to them. Hence, for different packings, the same item may have different weights. Do we gain from this relaxation in the sense of decreasing the PoA? Applying the proof of Theorem 4, the PoA cannot go below $4 / 3$, no matter how the weights are given, because no item can move in that construction. But possibly the $P o A$ can go below our bound $16 / 11 \approx 1.4545$. There are no results available for this version so far.

\section{Acknowledgements}

Gyorgy Dosa acknowledges the financial support of Széchenyi 2020 under the EFOP-3.6.1-16-2016-00015. Research of the first and third authors was supported in part by the National Research, Development and Innovation Office NKFIH under the grant SNN 116095.

\section{References}

[1] V. Bilò. On the packing of selfish items. In Proc. of the 20th International Parallel and Distributed Processing Symposium (IPDPS'06). IEEE, 2006. 9 pages.

[2] X. Chen, Q. Nong, and Q. Fang. An improved mechanism for selfish bin packing. In: Gao X., Du H., Han M. (eds) Combinatorial Optimization and Applications. COCOA 2017. Lecture Notes in Computer Science, vol 10627, pages 241-257, Springer, Cham.

[3] E. G. Coffman, Jr., J. Csirik, G. Galambos, S. Martello, and D. Vigo. Bin packing approximation algorithms: Survey and classification. In Handbook of Combinatorial Optimization, 455-531, Springer, New York, 2013.

[4] G. Dosa and L. Epstein. Generalized selfish bin packing. arXiv:1202.4080, 43 pages, 2012.

[5] G. Dosa, H. Kellerer, and Zs. Tuza. Bin Packing Games with Weight Decision: how to get a small value for the Price of Anarchy. In 16th Workshop on Approximation and Online Algorithms, WAOA 2018, Lecture Notes in Computer Science, to appear.

[6] G. Dosa and J. Sgall. First Fit bin packing: A tight analysis. In Proc. 30th International Symposium on Theoretical Aspects of Computer Science (STACS'13), 538-549. 
[7] G. Dosa and J. Sgall. Optimal analysis of best fit bin packing. Lecture Notes in Computer Science 8572, 429-471, 2014.

[8] L. Epstein and E. Kleiman. Selfish bin packing. Algorithmica, 60(2):368394, 2011.

[9] L. Epstein, E. Kleiman, and J. Mestre. Parametric packing of selfish items and the subset sum algorithm. In Proc. 5th International Workshop on Internet and Network Economics (WINE'09), 67-78, 2009.

[10] L. Epstein, E. Kleiman, and J. Mestre. Parametric packing of selfish items and the subset sum algorithm. Algorithmica, 74(1), 177-207, 2016.

[11] D. S. Johnson, A. Demers, J. D. Ullman, M. R. Garey, and R. L. Graham. Worst-case performance bounds for simple one-dimensional packing algorithms. SIAM J. Comput. 3, 299-325, 1974.

[12] E. Koutsoupias and C. H. Papadimitriou. Worst-case equilibria. In Proc. 16th Annual Symposium on Theoretical Aspects of Computer Science (STACS'99), 404-413, 1999.

[13] R. Ma, G. Dosa, X. Han, H.-F. Ting, D. Ye, and Y. Zhang. A note on a selfish bin packing problem. Journal of Global Optimization, 56(4), 14571462, 2013.

[14] Q. Q. Nong, T. Sun, T. C. E. Cheng, and Q. Z. Fang. Bin packing game with a price of anarchy of $\frac{3}{2}$. Journal of Combinatorial Optimization, 35, 632-640, 2018.

[15] Z. Wang, X. Han, G. Dosa, and Zs. Tuza. A general bin packing game: Interest taken into account. Algorithmica, 80(5), 1534-1555, 2018.

[16] G. Yu and G. Zhang. Bin packing of selfish items. In Proc. 4th International Workshop on Internet and Network Economics (WINE'08), 446-453, 2008.

[17] C. Zhang, G. Zhang. Cost-sharing mechanisms for selfish bin packing. In: Gao X., Du H., Han M. (eds) Combinatorial Optimization and Applications. COCOA 2017. Lecture Notes in Computer Science, vol 10627, pages 355-368, Springer, Cham. 